\title{
The Effectiveness of Eccentric-Concentric Training, Isometric Contractions, Scapular and Rotator Cuff Strengthening on Pain and Disability in Lateral Elbow Tendinopathy: A Case Report
}

\author{
Stasinopoulos Dimitrios* \\ Department of Health Sciences, European University of Cyprus, Cyprus
}

Submission: July 18, 2018; Published: July 26, 2018

*Corresponding author: Stasinopoulos Dimitrios, Department of Health Sciences, European University of Cyprus, Cyprus, Tel: 35722713044; Email: D.Stassinopoulos@euc.ac.cy

\section{Abstract}

Objective of the Study: The aim of the present case report is to present the effect of an exercise programme consisting of static stretching exercises, isometric, concentric-eccentric training and strengthening of rotator cuff and scapula muscles exercises on pain and disability in a patient experiencing Lateral Elbow tendinopathy (LET).

Case Report: A patient with unilateral LET for 6 months was included in the present report. The patient followed a supervised exercise programme consisting of slow progressive concentric-eccentric training of the wrist extensors, isometric training of the wrist extensors and static stretching exercises of the extensors muscles of the wrist five times per week for 4 weeks. In addition, the rotator cuff and scapular muscles were strengthened. The programme was individualized on the basis of the patient's description of pain experienced during the procedure. Outcome measures were pain, using a visual analogue scale, and function, using a visual analogue scale and the pain-free grip strength. The patients were evaluated at baseline, at the end of treatment (week 4) and 1 month (week 8) after the end of treatment.

Results: At the end of the treatment and at the follow-up there was a decline in pain and a rise in function.

Conclusion: The results of the present trial suggest that the combination of concentric-eccentric training of extensors of the wrist combined with isometric training of extensors, static stretching exercises of wrist extensors, strengthening of rotator cuff and scapular muscles can produce significant improvements in terms of pain and disability in LET.

\section{Introduction}

Lateral elbow tendinopathy (LET) seems to be the most appropriate term to use in clinical practice because all the other terms such as lateral epicondylitis, lateral epicondylalgia, lateral epicondylosis and/or tennis elbow refer to inappropriate aetiological, anatomical and pathophysiological terms [1]. LET is one of the most common lesions of the arm work-related or sport-related pain disorder. The condition is usually defined as a syndrome of pain in the area of the lateral epicondyle [2] that may be degenerative or failed healing tendon response rather than inflammatory [3]. Hence, the increased presence of fibroblasts, vascular hyperplasia, proteoglycans and glycosaminoglycans together with disorganized and immature collagen may all take place in the absence of inflammatory cells [4]. The most commonly affected structure is the origin of the extensor carpi radialis brevis (ECRB) [4]. The dominant arm is commonly affected, the peak prevalence of LET is between 30 and 60 years of age $[2,5]$ and the disorder appears to be of longer duration and severity in women $[3,6]$. The main complaints of patients with LET are pain and decreased function [2,3] both of which may affect daily activities. Diagnosis is simple, and a therapist should be able to reproduce this pain in at least one of three ways:

a) Digital palpation on the facet of the lateral epicondyle,

b) Resisted wrist extension and/or resisted middle-finger extension with the elbow in extension,

c) By getting the patient to grip an object $[2,3,5]$.

Although the signs and symptoms of LET are clear and its diagnosis is easy, to date, no ideal treatment has emerged. Many clinicians advocate a conservative approach as the treatment of choice for LET $[2,3,7,8]$. Physiotherapy is a conservative treatment that is usually recommended for LET patients [2,3,9]. A 
wide array of physiotherapy treatments has been recommended for the management of LET [10-14]. These treatments have different theoretical mechanisms of action, but all have the same aim, to reduce pain and improve function. Such a variety of treatment options suggests that the optimal treatment strategy is not known, and more research is needed to discover the most effective treatment in patients with LET [10-14].

One of the most common physiotherapy treatments for LET is an exercise programme $[2,3,9-14]$. One consisting of eccentric exercises has shown good clinical results in LET [15] as well as in conditions like LET in clinical behavior and histopathological appearance, such as patellar and Achilles tendinopathy [16]. Such an exercise programme is used as the first treatment option for our patients with LET $[9,17]$. Malliaras and his colleagues [16] concluded that clinicians should consider eccentric-concentric loading alongside or instead of eccentric loading in tendinopathy. Recently, isometric exercises are indicated to reduce and manage tendon pain [18-20]. LET is often related to forceful grip activities requiring isometric contraction of the wrist flexors and extensors [21]. Perhaps isometric contractions would be more beneficial than eccentric ones in lateral epicondylitis [21]. A recently clinical trial showed that the combination of concentric - eccentric training with isometric contraction had positive effects in LET [22].

Based mainly on clinical experience, rotator cuff and scapular musculature weakness in LET patients is commonly addressed increasing pain, decreasing functional ability and hand grip strength [23]. To our knowledge, there have been no studies, only a research protocol [24] to investigate the effectiveness of rotator cuff and scapular muscle strengthening in the management of LET. It is possible to combine, static stretching exercises, isometric and concentric-eccentric training with strengthening of rotator cuff and scapula muscles exercises for the management of LET. Therefore, the aim of the present case report is to present the effect of an exercise programme consisting of static stretching exercises, isometric, concentriceccentric training and strengthening of rotator cuff and scapula muscles exercises on pain and disability in a patient experiencing LET.

\section{Case Report}

\section{History}

Mr. A, a 47-year-old male who worked in pc about ten hours per day, complained of pain in the lateral aspect of his left elbow, of his non-dominant hand and with his finger pointed the site of pain, which was about three cm distally to the lateral epicondyle of the humerus on the facet of the lateral epicondyle. The pain was mainly there but sometimes spread down until the middle of the forearm. He was not able to describe any particular movements to explain why the pain spread down until that point. At the beginning, he experienced pain after working, which he could tolerate. Later, he had pain during his activity, which he could not tolerate and had to stop his job. Once he had stopped, the pain subsided within one hour. He visited his GP, who prescribed NSAIDs. He had a little improvement and his GP referred him to a course of physiotherapy. He had this kind of pain for about six months. He could sleep, but sometimes he felt a mild pain in gripping objects. During gripping, he was able to tolerate the pain. He did not complain of crepitus, stiffness, paresthesia, swelling, locking or cervical pain. He did not have any previous problems in the spine or the peripheral joints. He did not have cancer, diabetes or epilepsy and none in his family did. He did not have any operation in the past and did not take any medications at the time of assessment.

\section{Examination Findings}

His face, posture and gait were noted in observation. He was calm and slept without having any pain disturbing him. His posture as well as his gait was normal. The overall posture was assessed in search of body deformity. The position of the head, the cervical lordosis and the position of both shoulders were normal. The carrying angle was normal in comparison with the other side and there were not color changes, muscles wasting or swelling. Signs of inflammatory activity like heat, swelling and synovial thickening were not found. The movements of the neck and shoulder were pain free, with full range of motion and full power. The movements of the elbow joint, which were tested, were flexion and extension both passively and under resistance. The passive movements were pain free with full range of motion and normal end feel. The resisted movements were pain free with full power, meaning 5 on the Oxford scale. The movements of the proximal radioulnar, which were tested, were supination and pronation. These movements were tested both passively and under resistance. The passive movements were pain free with full range of motion and normal end feel and the resisted movements were pain free with full power, meaning 5 on the Oxford scale.

The movements of the wrist joint which were tested were flexion and extension. These movements were tested both passively and under resistance.

a) Passive Movements: The extension of the wrist with the elbow in extension was pain free, with full range of motion and normal end feel. The wrist flexion with the elbow in extension was slightly painful on the facet of the lateral epicondyle, but it involved full range of motion with normal end feel.

b) Resisted Movements: The flexion of the wrist with the elbow in extension was pain free and with normal power, meaning 5 on the Oxford scale. In the extension of the wrist with the elbow in extension there was pain over the facet of the lateral epicondyle of the humerus, 6/10 on the VAS and the power was 4 on the Oxford scale. Resisted extension of the middle finger was painful (5/10 on the VAS) on the facet and the power was 4 on the Oxford scale. The patient reported 
pain (6/10 on the VAS) with the handgrip dynamometer test. There was pain over the common extensor tendon on the facet of the lateral epicondyle of the humerus by palpation.

\section{Procedure}

The patient followed a supervised exercise programme consisting of slow progressive concentric-eccentric training of the wrist extensors, isometric training of the wrist extensors and static stretching exercises of the extensors muscles of the wrist. In the eccentric-concentric training combined with isometric contraction group the elbow was on the bed in full extension, the forearm in pronation, the wrist in an extended position (as high as possible), and the hand hanging over the edge of the bed [22]. From this position, patients flexed their wrist slowly while counting to 30 , then returned to the starting position (extension) [22]. In the starting position, the patient performed an isometric contraction of wrist extensors for 45 seconds [22]. When the isometric contraction completed the patient performed the eccentric-concentric contraction and so on [22].

Three sets of 15 repetitions of slow progressive exercises (eccentric, concentric and isometric respectively) of the wrist extensors at each treatment session were performed, with 1-min rest interval between each set. Patients were told to continue with the exercise even if they experienced mild pain. However, they were told to stop the exercise if the pain became disabling. The mild and disabling pain was monitored asking the patient to rate the pain on VAS before and after treatment. Mild pain was defined below 4 on VAS whereas disabling pain was defines above 8 on VAS $[25,26]$. When patients were able to perform the exercises without experiencing any minor pain or discomfort, the load was increased using free weights. The static stretching exercises were repeated six times at each treatment session, three times before and three times after the exercises, with a 30 second rest interval between each repetition. Static stretching exercises of the wrist extensors were performed with the help of the other hand. The patient's elbow was placed in full extension, the forearm in full pronation, and the wrist in flexion and ulnar deviation according to the patient's tolerance. This position was held for 30-45 seconds each time and then released [25-27]. In addition, the rotator cuff and scapular muscles were strengthened. The strengthening exercises was included:

a) Shoulder medial and lateral rotation with the elbow in 0 and 90 degrees of abduction

b) Shoulder abduction to 90 degrees with elbow in flexion

c) Scaption-the arm was kept at 30 degrees of horizontal abduction with the thumb pointing downwards

d) Diagonal pattern from full flexion to extension [28].

Scapular muscle such as Rhomboids, upper trapezius, levator scapulae and serratus anterior were also strengthened in the present protocol [29]. Each exercise was performed twice at each treatment session with 12 repetitions in each set and 1 min rest interval between each set. Patients were told to continue with the exercise even if they experienced mild pain. However, they would be told to stop the exercise if the pain became disabling (definition of mild and disabling pain please see above). When patients were able to perform the strengthening exercises without experiencing any minor pain or discomfort, the load was increased using free weights or Thera bands. Supervised exercise programme was given five times a week for 4 weeks and was individualized on the basis of the patient's description of pain experienced during the procedure. The patient was instructed to use her arm during the course of the study but to avoid activities that irritated the elbow such as grasping, lifting, knitting, handwriting, driving a car and using a screwdriver. She was also told to refrain from taking anti-inflammatory drugs throughout the course of the study. Patient compliance with this request was monitored using a treatment diary.

Communication and interaction (verbal and non-verbal) between the therapist and patient was kept to a minimum, and behaviors sometimes used by therapists to facilitate positive treatment outcomes were purposefully avoided. For example, patients were given no indication of the potentially beneficial effects of the treatments or any feedback on their performance in the pre-application and post application measurements [30]. Pain and function were measured in the present study. The patient was evaluated at the baseline (week 0), at the end of treatment (week 4) and at 1 month (week 8) after the end of treatment. Pain was measured on a visual analogue scale (VAS), where $0 \mathrm{~cm}$ was "least pain imaginable" and $10 \mathrm{~cm}$ was "worst pain imaginable". The pain VAS was used to measure the patient's worst level of pain over the previous $24 \mathrm{~h}$ before each evaluation, and this approach has been shown to be valid and sensitive of the VAS [31].

Function was measured using a VAS, in which $0 \mathrm{~cm}$ was taken as "no function" and $10 \mathrm{~cm}$ as "full function". Patients were instructed to report their overall level of elbow function over the previous $24 \mathrm{~h}$ before each evaluation, and this approach has been shown to be valid and sensitive of the VAS [31]. In addition, function was measured by pain-free grip strength. Pain-free grip strength is defined as the amount of force each patient can generate with an isometric gripping action before eliciting pain [30] Force was measured in pounds with a Jamar hand dynamometer that had adjustable handles to accommodate different hand sizes. The arm was placed in a standardized position of elbow extension, forearm pronation and internal rotation of the upper limb such that the palmar aspect of the hand faced posteriorly with the upper limb placed by the patient's side. Patient was then instructed to squeeze the dynamometer handles until she first experienced pain and then to release her grip [30]. The attained grip force was subsequently recorded, and the reading was not visible to the patient. Three measures of pain-free grip strength were recorded with a $30 \mathrm{~s}$ rest interval between each measurement, and the mean value of these repetitions was calculated. 


\section{Results}

Pain on VAS was 9, function on VAS was 3 and pain-free grip strength was $24 \mathrm{lb}$ at the initial evaluation. At the end of the treatment (week 4), there was a decline in pain on VAS of 6 units, a rise in function on VAS of 5 units and a rise in pain-free grip strength of 38 units. At week 8, the pain on VAS was 1, function on VAS was 9 and the pain-free grip strength was 68lb (Table 1).

Table 1: Pain, function and pain-free grip strength over the $24 \mathrm{~h}$ before each evaluation.

\begin{tabular}{|l|c|c|c|}
\hline & Pain (cm) & $\begin{array}{c}\text { Function } \\
\text { (cm) }\end{array}$ & $\begin{array}{c}\text { Pain-free grip strength } \\
\text { (lb) }\end{array}$ \\
\hline WEEK 0 & 9 & 3 & 24 \\
\hline WEEK 4 & 3 & 8 & 62 \\
\hline WEEK 8 & 1 & 9 & 68 \\
\hline
\end{tabular}

\section{Discussion}

The present study has looked at the effect of concentriceccentric training of extensors of the wrist combined with isometric training of extensors, static stretching exercises of wrist extensors, strengthening of rotator cuff and scapular muscles in a patient experiencing LET and its findings have demonstrated significant improvements in terms of pain and disability. The results obtained from this case report are novel; as to date, similar studies have not been conducted. Alfredson et al. [32] were first proposed the eccentric training of the injured tendon. It is the most commonly used conservative approach in the treatment of tendinopathy. Malliaras and his colleagues [16] concluded that clinicians should consider eccentric-concentric loading alongside or instead of eccentric loading in Achilles and patellar tendinopathy. A Heavy Slow Resistance (HSR) program is recommended in the management of lower limb tendinopathy $[33,34]$. The HSR program was produced equivalent pain and function improvement (VISA) than the Alfredson eccentric program, but significantly better patient satisfaction at six months follow-up. In the Achilles tendon, eccentric and HSR have recently been shown to yield similar clinical outcomes (VISA and patient satisfaction) at 1 year follow up. Based on the above findings, the HSR program can be recommended as an alternative to the Alfredson eccentric program lower limb tendinopathy rehabilitation. Studies determining the effectiveness of such as exercises at other tendinopathies such as LET are needed.

Systematic review [15] and RCT [35] favor eccentric over other types of contractions in the management of LET, but Martinez-Silvestrini et al. [21] stated that, unlike Achilles tendinopathy, LET is often related to forceful grip activities requiring isometric contraction, which would be more beneficial than eccentric contraction in LET. Recently, isometric exercises have been recommended to reduce and manage tendon pain increasing the strength at the angle of contraction without producing inflammatory signs [16]. Five repetitions of 45-second isometric mid-range quadriceps exercise at $70 \%$ of maximal voluntary contraction have been shown to reduce patellar tendon pain for 45 minutes post exercise and this was also associated with a reduction in motor cortex inhibition of the quadriceps that was associated with patellar tendinopathy [18]. The dosage of isometric contractions in the present was based on clinical experience [18-20]. and their effect on pain in patients with LET requires further study. Therefore, it was hypothesized that the simultaneous use of these two kinds of contractions (isotonic and isometric) and static stretching exercises will further enhance the analgesic effect of contractions in the treatment of LET, increasing the arm function.

It has also been proposed that the positive effects of exercise programmes for tendon injuries may also be attributable to the effect of stretching. The aim of stretching is to lengthen the muscle-tendon unit, orientate the new collagen fibres and experience consequently less strain during joint motion [36]. Stretching may increase the range of motion of the relevant joint and strengthen the tendon or make it more resistant to strain (Silva et al., 2016). Based mainly on clinical experience, rotator cuff and scapular musculature weakness in LET patients is commonly addressed increasing pain, decreasing functional ability and hand-grip strength [23]. This suggests that the underlying causes of LET may not be restricted to the elbow region [23]. Functional impingement of the shoulder due to altered joint mechanism and muscle imbalance can impair the stabilization of the shoulder resulting in overcompensation of the extensors of the wrist [3]. This may lead to micro trauma of the soft tissue structures present at the lateral epicondyle thus causing symptoms of LET [3]. It is plausible that improvements with gripping might have occurred from a combination of improved motor control of the scapula and improved muscular strength of the scapular musculature [29]. Changes in the shoulder girdle may lead to altered and compensatory changes in the forearm and hand which may overload the muscles of the forearm during repetitive movements, thus causing symptoms of LET [3]. Using corrective scapular exercises and rotator cuff strengthening exercises, normal motion might have been restored, resulting in resolution of pain with activities and a return to pain-free gripping for the patient [29]. On the other hand, there are no studies to support this belief.

The load of exercises was increased according to the patients' symptoms otherwise the results are poor [37]. Furthermore, eccentric exercises were performed at a low speed in every treatment session because this allows tissue healing [4]. Ice was recommended at the end of the treatment, but research has shown that ice as a supplement to an eccentric exercise programme offers no benefit to patients with tendinopathy [26]. Finally, the avoidance of painful activities is crucial for tendon healing, because training during the treatment period increases patients' symptoms and delays tendon healing [38]. Eccentric exercises appear to reduce the pain and improve function, reversing the pathology of LET $[27,37,39,40]$ as supported by experimental studies on animals [41-44]. The way that eccentric training achieves the goals remains uncertain, as there is a lack of good quality evidence to confirm that physiological effects 
translate into clinically meaningful outcomes and vice versa. The clinical improvement of the HSR group was accompanied by increased collagen turnover. It is unknown if the isometric contractions can reverse the pathology of the tendinopathy and in this case the pathology of LET or reduce only the pain.

Although a home exercise programme can be performed any time during the day without requiring supervision from a therapist, our clinical experience has shown that patients fail to comply with the regimen of home exercise programmes [26]. Although many ways can be recommended to improve the compliance of patients with the home exercise programme such as phone calls, exercise monitors and better self-management education, it is believed that this problem can be really solved by the supervised exercise programmes performed in a clinical setting under the supervision of a therapist. It is believed because our experience has shown that many patients stopped the home exercise programme without giving explanations, whereas patients completed the supervised programme. One possible reason why they continue the supervised exercise programme could be the cost. In the supervised exercise programme, the patients visit the therapist more times than the home exercise programme, and this is more expensive. A future study will combine the both types of exercise programmes in order to maximize the compliance of the patients.

Even though the positive effects of such an exercise programme in LET have been reported in the present report, its study design limits the generalization of these findings. Future well-designed clinical trials are needed to confirm the positive results of this case study establishing the effectiveness of such an exercise program in the management of LET. In addition, structural changes in the tendons related to the treatment interventions and the long-term effects (6 months or more after the end of treatment) of these treatments are needed to investigate. Further research is needed to establish the possible mechanism of action of this treatment approach, and the cost effectiveness of such treatment, because reduced cost is an important issue for the recommendation of any given treatment.

\section{Conclusion}

The exercise programme, consisting of concentric-eccentric, static stretching and isometric exercises of the wrist extensors, strengthening of rotator cuff and scapular muscles had reduced the pain and improved the function in a patient with LET at the end of the treatment and at one-month follow-up. Further welldesigned trials are needed to confirm the results of the present case report.

\section{References}

1. Stasinopoulos D, Johnson MI (2006) Lateral elbow tendinopathy is the most appropriate diagnostic term for the condition commonly referred to as lateral epicondylitis. Medical Hypotheses 67(6): 1400-1402.

2. Bisset L, Vicenzino B (2015) Physiotherapy management of lateral epicondylalgia. J Physiothe 61(4): 174-181.
3. Coombes B, Bisset L, Vicenzino B (2015) Management of Lateral Elbow Tendinopathy-One Size Does Not Fit All. J Orthop Sports Phys Ther 45(11): 938-949.

4. Kraushaar B, Nirschl R (1999) Current concepts review - tendinosis of the elbow (tennis elbow). Clinical features and findings of histological immunohistochemical and electron microscopy studies. J Bone Joint Surg Am 81(2): 259-278.

5. Vicenzino B, Wright A 1996) Lateral epicondylalgia I: Epidemiology, pathophysiology, aetiology and natural history. Phys Ther Rev 1(1): 23-34.

6. Waugh E, Jaglal S, Davis A, Tomlinson G, Verrier MC, et al. (2004) Factors associated with prognosis of lateral epicondylitis after 8 weeks of physical therapy. Arch Phys Med Rehabil 85(2): 308-318.

7. Haker E (1993) Lateral epicondylalgia: Diagnosis, treatment and evaluation. Crit Rev Phys Rehabil Med 5: 129-154.

8. Hong Q, Durand M, Loisel P, (2004) Treatment of lateral epicondylitis where is the evidence? Joint Bone Spine 71(5): 369-373.

9. Stasinopoulos D, Johnson MI (2004) Physiotherapy and tennis elbow/ lateral epicondylitis. Letter Tennis elbow: BMJ 327: 329.

10. Trudel D, Duley J, Zastrow I, Kerr EW, Davidson R, et al. (2004) Rehabilitations for patients with lateral epicondylitis: A systematic review. J Hand Ther 17(2): 243-66.

11. Smidt N, Assendelft W, Arola H, Malmivaara A, Greens S, et al. (2003) Effectiveness of physiotherapy for lateral epicondylitis: A systematic review. Ann Med 35(1): 51-62.

12. Labelle H, Guibert R, Joncas J, Newman N, Fallaha M, et al. (1992) Lack of scientific evidence for the treatment of lateral epicondylitis of the elbow: An attempted meta-analysis. J Bone Joint Surg 74(5): 646-651.

13. Wright A, Vicenzino B (1997) Lateral epicondylalgia II: therapeutic management. Phys Ther Rev 2(1): 39-48.

14. Bisset L, Paungmali A, Vicenzino B (2005) A systematic review and meta-analysis of clinical trials on physical interventions for lateral epicondylalgia. British Journal of Sports Medicine 39(7): 411-422.

15. Raman J, MacDermid JC, Grewal R (2012) Effectiveness of different methods of resistance exercises in lateral epicondylosis-a systematic review. J Hand Ther 25(1): 5-25.

16. Malliaras P, Barton C, Reeves N (2013) Achilles and patellar tendinopathy loading programmes: A systematic review comparing clinical outcomes and identifying potential mechanisms for effectiveness. Sports Med 43(4): 267-286.

17. Stasinopoulos D, Johnson MI (2004) Treatment/management for tendinopathy. Time to abandon the 'tendinitis' myth. BMJ 324: 626.

18. Rio E, Kidgell D, Purdam C, Gaida J, Moseley GL, et al. (2015) Isometric exercise induces analgesia and reduces inhibition in patellar tendinopathy. Br J Sports Med 49(19): 1277-1283.

19. Stasinopoulos D (2015) The Effectiveness of Isometric Contractions Combined with Eccentric Contractions and Stretching Exercises on Pain and Disability in Lateral Elbow Tendinopathy. A case Report. Journal of Novel Physiotherapies 5: 1-5.

20. Young Park J, Park H, Choi J, Sun M, Soo K, et al. (2010) Prospective evaluation of the effectiveness of a home based program of isometric strengthening exercises: A 12 month follow up. Clinics in orthopaedic surgery 2(3): 173-178.

21. Martinez-Silvestrini JA, Newcomer KL, Gay RE, Schaefer MP, Kortebein P, et al. (2005) Chronic lateral epicondylitis: comparative effectiveness of a home exercise program including stretching alone versus stretching supplemented with eccentric or concentric strengthening. J Hand Ther 18(4): 411-419. 
22. Stasinopoulos D, Stasinopoulos I (2017) Comparison of effects of eccentric training, eccentric-concentric training and eccentricconcentric training combined with isometric contraction in the treatment of Lateral Elbow Tendinopathy. Journal of hand therapy $30(1): 13-19$.

23. Day JM (2015) Scapular muscle performance in individuals with lateral epicondylalgia. Journal of Orthopaedic and Sports Physical Therapy 45(5): 414-424

24. Stasinopoulos D, Constantinou A, Cheimonidou AZ, Lamnisos D (2017) There is benefit to strengthen the rotator cuff and scapular muscles in patients with lateral elbow tendinopathy? A research protocol EC Orthopaedics 6(4): 155-162.

25. Stasinopoulos D, Stasinopoulos I (2006) Comparison of effects of cyriax physiotherapy, a supervised exercise and polarized polychromatic non-coherent light (Bioptron light) for the treatment of latera epicondylitis. Clin. Rehabil 20(1): 12-23

26. Manias P, Stasinopoulos D (2006) A controlled clinical pilot trial to study the effectiveness of ice as a supplement to the exercise programme for the management of lateral elbow tendinopathy. British Journal of Sports Medicine 40(1): 81-85.

27. Stasinopoulos D, Stasinopoulos I, Manias P (2010) Comparison of effects of a home exercise programme and a supervised exercise programme for the management of lateral elbow tendinopathy, British Journal of Sports Medicine 44(8): 579-583.

28. Bhatt JB, (2013) Middle and lower trapezius strengthening for the management of lateral epicondylalgia: a case report. Journal of Orthopaedic and Sports Physical Therapy 43(11): 841-847.

29. Sharma M, Eapen C, Kamath J (2015) Effect of adding rotator cuff strengthening to therapeutic ultrasound and wrist extensor eccentric exercise for lateral epicondylalgia-A randomized clinical trial. Int J Health Sci Res 5(7): 250-257.

30. Vicenzino B, Collins D, Wright A (1996) The initials effects of cervical spine manipulative physiotherapy treatment on the pain and dysfunction of lateral epicondylalgia Pain 68(1): 69-74.

31. Stratford P (1987) Extensor carpi radialis tendonitis: a validation of selected outcome measures. Physiotherapy Canada 39(4): 250-255.

32. Alfredson H, Pietila T, Johnson P (1998) Heavy load eccentric calf muscle training for the treatment of chronic Achilles tendinosis. Am J Sports Med 26(3): 360-366.

33. Beyer R, Kongsgaard M, Hougs Kjaer B, Kjaer M, Magnusson SP, et al. (2015) Heavy Slow Resistance Versus Eccentric Training as Treatment for Achilles Tendinopathy: A Randomized Controlled Trial. Am J Sports Med 43(7): 1704-1711.

34. Kongsgaard M, Kovanen V, Aagaard P, Doessing S, Hansen P, et al. (2009) Corticosteroid injections, eccentric decline squat training and heavy slow resistance training in patellar tendinopathy. Scandinavian Journal of Medicine \& Science in Sports 19(6): 790-802.

35. Peterson M, Butler S, Eriksson M (2014) A randomized controlled trial of eccentric vs. concentric graded exercise in chronic tennis elbow (lateral elbow tendinopathy). Clin Rehabil 28(9): 862-872.

36. Stanish W, Rubinovich R, Curwin S, (1986) Eccentric training in chronic tendinitis. Clin Orthopaedics Relat Res 208: 65-68.

37. Jensen K, Di Fabio R (1989) Evaluation of eccentric exercise in treatment of patellar tendinitis. Physical Therapy 69(3): 211-216.

38. Visnes H, Cook J, Bahr R (2005) No effect of eccentric training on Jumper's knee in volleyball players during the competitive season. A randomized clinical trial. Clin J Sports Med 15(4): 227-234.

39. Hawary R, Stanish W, Curwin S (1997) Rehabilitation of tendon injuries in sport. Sports Med 24(5): 347-358.

40. Khan K, Cook J, Kannus P (2002) Time to abandon the "tendonitis" myth. BMJ 324(7338): 626-627.

41. Khan K, Cook J, Taunton J (2000) Overuse tendinosis, not tendinitis: A new paradigm for a difficult clinical problem. Phys Sportsmed 28(5): 38-48.

42. Malliaras P, Cook J, Purdam C (2015) Patellar Tendinopathy: Clinical Diagnosis, Load Management, and Advice for Challenging Case Presentations. J Orthop Sports Phys Ther 45(11): 887-898.

43. Scattone Silva R, Nakagawa TH, Ferreira AL, Garcia LC, Santos JE, et al. (2016) Lower limb strength and flexibility in athletes with and without patellar tendinopathy. Physical Therapy in Sport 20: 19-25.

44. Stasinopoulos D, Stasinopoulos I, Stasinopoulou K (2014) A pilot tria to study the effectiveness of an exercise programme in the treatment of rotator cuff tendinopathy. Biology of exercise 10(2): 69-77

\section{Your next submission with Juniper Publishers will reach you the below assets}

- Quality Editorial service

- Swift Peer Review

- Reprints availability

- E-prints Service

- Manuscript Podcast for convenient understanding

- Global attainment for your research

- Manuscript accessibility in different formats ( Pdf, E-pub, Full Text, Audio)

- Unceasing customer service

\section{Track the below URL for one-step submission}

https://juniperpublishers.com/online-submission.php 